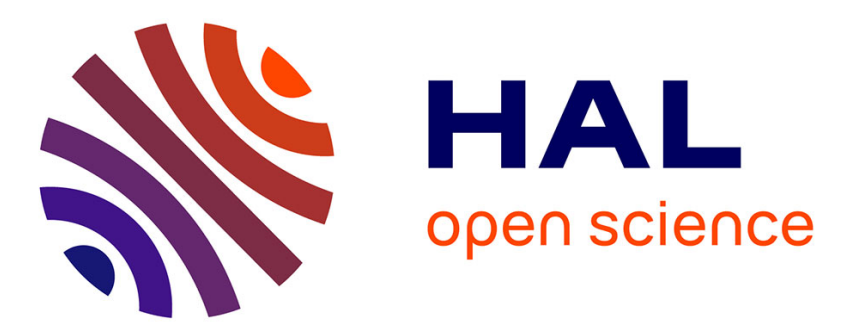

\title{
Romain Gaignard et la Pampa argentine : le regard original et perspicace d'un Européen
}

Rubén Devoto, Martine Guibert

\section{To cite this version:}

Rubén Devoto, Martine Guibert. Romain Gaignard et la Pampa argentine: le regard original et perspicace d'un Européen. Caravelle. Cahiers du monde hispanique et luso-brésilien, 2002, 79, pp.219224. halshs-00703733v2

\section{HAL Id: halshs-00703733 \\ https://shs.hal.science/halshs-00703733v2}

Submitted on 9 Jul 2012

HAL is a multi-disciplinary open access archive for the deposit and dissemination of scientific research documents, whether they are published or not. The documents may come from teaching and research institutions in France or abroad, or from public or private research centers.
L'archive ouverte pluridisciplinaire HAL, est destinée au dépôt et à la diffusion de documents scientifiques de niveau recherche, publiés ou non, émanant des établissements d'enseignement et de recherche français ou étrangers, des laboratoires publics ou privés. 


\title{
Romain Gaignard et la Pampa argentine : le regard original et perspicace d'un Européen
}

\author{
PAR \\ Rubén DEVOTO \\ INTA, Buenos Aires \\ Martine GUIBERT \\ Dynamiques rurales, Université de Toulouse-Le Mirail
}

\section{Liminaire}

A la fois modeste témoignage et occasion de reconnaissance, ce bref article propose de jeter un regard sur le devenir de la Pampa argentine, dans le sillage de Romain Gaignard et des travaux qu'il a réalisés à partir des années 1960. Ses analyses, éclairantes et originales dans le contexte argentin de l'époque, marient approche historique et réflexion prospective, et restent une base solide pour avancer l'hypothèse selon laquelle une place reste réservée à l'Argentine agricole et agro-alimentaire sur l'échiquier mondial des grands producteurs et exportateurs, si tant est que le lien privilégié avec l'Europe est renouvelé, à travers de nouveaux produits agricoles tels les oléagineux et leurs dérivés.

\section{Un regard international}

Au milieu des années 1960, quand Romain Gaignard se trouve confronté à l'Argentine et à la région pampéenne et les retient comme objets de ses réflexions, un débat virulent se développe dans le pays. Depuis 1940, un grand nombre de producteurs délaissent le blé, le maïs et le lin, les trois cultures qui ont été à l'origine de la mise en valeur de millions d'hectares de la Pampa à partir de la fin du XIXe siècle. La chute 
remarquable du niveau de production provoque, en corollaire, une diminution très significative des volumes exportés. Alors qu'entre 1934 et 1938, l'Argentine a fourni $19 \%$ du blé et de la farine de blé commercialisés sur le marché mondial, entre 1959 et 1962 cette proportion n'est plus que de $6 \%$. Entre les mêmes périodes, les ventes argentines de maïs représentent respectivement $64 \%$ et $19 \%$ du total des exportations mondiales. La production bovine vit elle aussi de grands changements car, à la même époque, du début des années 1940 au milieu des années 1950, l'élevage se substitue bien souvent aux activités agricoles. Cette recrudescence de l'élevage, cependant, ne se traduit pas par une plus grande entrée de devises puisque la viande est destinée au marché interne. L'industrialisation par substitution des importations a créé un marché urbain croissant pour la consommation de la viande bovine, dont les prix sont stabilisés par le gouvernement, dans le souci de ne pas perturber le pouvoir d'achat des classes moyennes et de celles récemment urbanisées.

En d'autres termes, les années postérieures à la Seconde guerre mondiale ont généré un scénario de rupture, celui du premier grand éloignement de l'Argentine des circuits internationaux des biens agricoles et alimentaires. Durant ces années, les Etats-Unis et, dans une moindre mesure, le Canada, l'Australie et la Nouvelle-Zélande se sont peu à peu approprié ces marchés.

Le débat national à propos de la stagnation de l'agriculture pampéenne balance, alors, entre deux courants de pensée : l'un désigne comme principaux coupables les propriétaires terriens et les estancieros, étant donné leur attitude de rentier, bien peu capitaliste ; l'autre estime que les politiques industrielles et "populistes" des gouvernements successifs vont à l'encontre du monde agro-pastoral avec le prélèvement d'impôts, des prix internes déprimés et le contrôle par l'Etat des exportations. Les travaux de Romain Gaignard, d'une certaine manière et étant donné l'objectif précis de sa recherche de thèse d'Etat (intitulée "la mise en valeur de la région pampéenne " et soutenue en 1979), paraissent se situer davantage du côté du premier groupe d'analystes que du côté du second groupe. Si l'on faisait, de plus, une rétrospective de ses amitiés en Argentine, celles-ci se situeraient, de manière privilégiée, dans ce premier groupe qui, politiquement, signale et critique l'attitude de rentier de "l'oligarchie des grands propriétaires terriens ", et pas dans le groupe plus affilié à l'élite propriétaire, intrinsèquement moins démocratique et en permanence disposée à apporter son soutien aux différents coups d'Etat militaires qui ont affecté la République argentine, surtout à partir de 1966.

Romain Gaignard a en effet réalisé une étude méticuleuse du processus d'appropriation de la terre, étude qu'aucun Argentin n'avait jusqu'alors menée à bien. Appuyés sur une documentation 
impressionnante, ses travaux consistent à cerner le comment et le pourquoi de l'origine des immenses latifundios pampéens, qui ancrent dans la plaine le patronyme de familles devenues, en peu de temps, propriétaires d'une formidable richesse immobilière difficile à calculer. Il a par ailleurs travaillé sur la petite colonisation agricole qui a prédéterminé le dualisme agraire caractérisant aussi les structures de production pampéennes. Par cette méthode de recherche, basée sur le rassemblement méticuleux de sources bibliographiques, certaines étant encore inconnues, les études rurales et les travaux de recherche, que ce soit dans le champ de l'histoire ou le champ de la géographie, ont effectué en Argentine un important saut qualitatif. Sans doute ses écrits laissent-ils transparaître l'étonnement français et européen (notre regard habitué aux petites parcelles, à la très petite propriété) face à l'immensité d'un territoire entre les mains de quelques-uns, d'une poignée de familles qui ont eu tant à voir avec le savoir-faire productif et politique national, depuis la fondation du pays jusqu'au XXe siècle même.

Ainsi, Romain Gaignard, en tant qu'étranger, échappe au réductionnisme régnant en Argentine. Il est en condition de comprendre avec plus d'impartialité la situation générale dans le pays et, par ailleurs, il peut porter un regard moins passionné et, par-dessus tout, moins "nombriliste " et plus international. Bien que ses travaux ne l'expriment pas directement, car ce n'est pas le coeur de son analyse, certaines parties égrènent des explications différentes de celles répandues dans le pays, et tentent d'ébaucher l'avenir possible pour l'Argentine, qui peut passer par une nouvelle insertion sur le marché européen des biens agricoles et alimentaires. De plus, en tant qu'Européen et défenseur de la Communauté économique européenne et de la PAC (Politique agricole commune), sa participation intellectuelle au débat laisse entendre que la détermination de l'Europe occidentale à atteindre l'auto-suffisance en viandes, céréales et produits laitiers est l'une des causes incontournables qui ont écarté l'Argentine des marchés agro-alimentaires internationaux. A ce propos, il est particulièrement étrange de constater que, dans la littérature politique et commerciale argentine de l'époque (années 1960 et 1970), les références à la CEE et à la PAC comme éléments néfastes aux agro-exportations argentines sont rares. Prédominent plutôt le regard interne et des disputes fermées et acerbes envers l'adversaire social et politique local. Or, dans les écrits de Romain Gaignard réside cette conviction d'une réinsertion possible de l'Argentine avec, à nouveau, l'Europe comme principale cliente de ses produits agro-pastoraux. L'Argentine se trouve alors " en retard " par rapport au Brésil qui, dès la deuxième moitié des années 1960 , exporte plus de 280000 tonnes de tourteaux de soja. Or, pour elle, la culture du soja est perçue pratiquement comme une excentricité et, pour les Argentins, il s'agit 
d'une aventure productive impossible ou inimaginable. Mais tel n'est pas le cas de Romain Gaignard.

\section{Le curieux retard}

"Una década de retraso... Es ya un extraño retraso! ": ainsi s'exprime Romain Gaignard à la fin des années 1970. Ce n'est en effet que dans les années 1980 (à partir de la récolte de 1982) qu'une nette réorientation productive se dessine dans la Pampa. Centrée sur le soja, elle entraîne de nouvelles configurations culturales dans la Pampa humide, où le soja est associé au blé. Au fil des décennies, il gagne les zones plus sèches, déplaçant les autres cultures et faisant reculer l'élevage vers I'Ouest. La fin du XXe siècle a ainsi vu la Pampa se couvrir de soja, en réaction à l'affirmation du marché européen, dépendant des approvisionnements internationaux en protéines végétales. Cette dépendance repose sur l'accord passé au lendemain de la Seconde guerre mondiale, et régulièrement reconduit, entre les Etats-Unis et l'Europe, qui stipule que celle-ci peut appliquer les techniques d'élevage intensif nord-américains à condition d'acquérir les tourteaux et graines de soja nécessaires sur le marché mondial, qui se résume alors aux Etats-Unis, avant de s'élargir au Brésil et à l'Argentine.

L'Argentine a finalement réagi au débouché européen constant, régulier et solvable, au point d'être à son tour en grande partie dépendante de ce marché quasiment captif. Sa production de soja, de l'ordre actuellement de 20 millions de tonnes, est exclusivement réservée à l'exportation (cas, aussi, d'une grande partie des 5 millions de tonnes de tournesol). Graines et tourteaux prennent le chemin, dans leur grande majorité, des usines de trituration et des élevages installés en Europe occidentale, l'huile étant écoulée sur les marchés asiatiques et moyensorientaux. Un quart des devises obtenues par les agro-exportations le sont grâce à la filière soja.

\section{Des producteurs aujourd'hui avant-gardistes}

Aujourd'hui, du fait de la dernière "révolution agricole " vécue par la Pampa à partir des années 1990 , cet étrange manque de réaction rapide paraît appartenir à un autre temps. La donne macro-économique renouvelée, basée sur une monnaie nationale à parité fixe avec le dollar, a bouleversé les paramètres financiers et fiscaux, et a créé une stabilité qui a déterminé de nouveaux comportements productifs et commerciaux, encouragés par des prix internationaux à ce moment-là revigorés.

A l'aube du XXI e siècle, et tout en ajustant leurs méthodes de production aux effets dévastateurs de la crise économique et politique actuelle, les producteurs argentins emploient des moyens de production 
et de commercialisation que les producteurs européens commencent à peine à appliquer, certains étant plus particulièrement destinés à préserver les potentiels agrologiques de la Pampa. Tandis que les contratistas, entreprises de service agricole, deviennent des acteurs incontournables du monde agricole pampéen, le semis direct, les semences génétiquement modifiées, la surveillance et le guidage par satellite sont des techniques de plus en plus courantes et, proportionnellement au nombre de producteurs, bien plus répandues qu'en Europe. De même, les marchés à terme transforment les opérations de cotations en bourse des lots de produits agricoles. Familiers aux producteurs pampéens, ils ne sont guère connus des agriculteurs européens, habitués aux rigidités administratives nationales et communautaires qui laissent peu de place à l'improvisation.

En d'autres termes, la souplesse d'action et de réaction des producteurs pampéens fait d'eux des "spéculateurs", au sens plein du terme. Ils conjuguent incertitudes commerciales et opportunités salutaires, instabilités macro-économiques et attentisme prudent, tout en étant désormais davantage conscients de la dégradation du potentiel productif de la Pampa et moins "prédateurs" que par le passé.

\section{Rapprocher les acteurs}

Depuis toujours convaincu de ces saisissantes capacités d'adaptation, Romain Gaignard n'en oublie pas pour autant de s'intéresser aux actions en amont et en aval de l'activité agricole, en portant son regard sur les acteurs agro-industriels, maillon indispensable des filières. Et, d'ailleurs, du fait des liens ténus établis avec l'Argentine et avec, notamment, des producteurs et des agro-industriels de la Pampa, et étant donné ses convictions pro-européennes, Romain Gaignard a naturellement endossé, au-delà de son rôle d'analyste universitaire, l'habit du professionnel. En participant activement avec les membres de l'interprofession au développement à l'international de la filière oléo-protéagineuse française, il a cuvré au rapprochement des acteurs du secteur de la transformation en pensant des partenariats industriels et commerciaux et en préconisant le développement de nouveaux produits (comme, actuellement, le "biodiesel " ou diesterer).

\section{Remarques conclusives}

A l'interface des mondes universitaire et agricole, Romain Gaignard tente de comprendre les réalités complexes du monde agricole pampéen et de l'Argentine, pays somme toute insaisissable de par ses crises politiques et économiques fréquentes, et qui semble se jouer des analyses, tout à tour sombres ou optimistes. En ce début de nouveau millénaire, 
ses analyses et sa méthode de recherche restent ô combien réalistes et pertinentes : c'est en ne désespérant pas de l'avenir, même lorsque le présent est décevant, et en n'oubliant pas certaines récurrences historiques, que l'objectivité du regard porté sur l'Argentine et sa Pampa est rendue possible. Attirés par sa méthode de travail et la rigueur de l'analyse des sources, de nombreux étudiants et jeunes chercheurs, argentins et européens, ont bénéficié de ses conseils et de ses réflexions, très souvent, aussi, de son énergie à décrocher des bourses et à mettre en place des accords de coopération durables. A ces chercheurs en développement local et rural, il a finalement transmis une sorte de posture scientifique dont le défi consiste à comprendre (et à faire comprendre) un processus depuis une perspective détachée et appuyée sur des sources incontestables, et à porter un regard qui dépasse les conflits locaux, internes et conjoncturels. Nous qui avons été ses étudiants espérons le revoir sur le terrain et nous croyons, de plus, que la Pampa argentine garde le désir secret que Romain Gaignard l'observe à nouveau, et recrée un espace interprétatif et prospectif distinct, avec son regard européen, original et perspicace.

RÉSUMÉ- En suivant la ligne de pensée ouverte par Romain Gaignard, il est permis d'avancer que l'avenir de la Pampa argentine passe par une adaptation permanente aux besoins changeants du marché européen. Le dynamisme de ses producteurs, malgré les crises et les difficultés, et à l'encontre d'une idée reçue,' peut être source d'inspiration pour les agriculteurs européens.

RESUMEN- De acuerdo a la línea iniciada por Romain Gaignard, se puede pensar que el porvenir de la pampa argentina quedará vinculado a una adaptación permanente a las necesidades variables del mercado europeo. A pesar de crisis y dificultades y contra el consabido tópico, el dinamismo de los productores pampeanos puede ser un modelo para los agricultores europeos.

ABSTRACT-Following Romain Gaignard's way of thinking, it is possible to assume that the argentinian Pampa's future has to go through a permanent adaptation to the changing needs of the european market. Its productors' dynamism, in spite of different crisis and difficulties, against a generally held belief, could be a source of inspiration for european farmers.

MOTS-CLES: Pampa argentine, Union européenne, Soja, Production agricole, Exportation. 